\title{
Creating Acoustic Maps Employing Supercomputing Cluster
}

\author{
Andrzej CZYŻEWSKI, Józef KOTUS, Maciej SZCZODRAK \\ Gdansk University of Technology \\ Multimedia Systems Department \\ Narutowicza 11/12, 80-233 Gdańsk, Poland \\ e-mail: ksm@sound.eti.pg.gda.pl \\ (received January 28, 2011; accepted February 24, 2011)
}

\begin{abstract}
The implemented online urban noise pollution monitoring system is presented with regard to its conceptual assumptions and technical realization. A concept of the noise source parameters dynamic assessment is introduced. The idea of noise modeling, based on noise emission characteristics and emission simulations, was developed and practically utilized in the system. Furthermore, the working system architecture and the data acquisition scheme are described. The method for increasing the speed of noise map calculation employing a supercomputer is explained. The practical implementation of noise maps generation and visualization system is presented, together with introduced improvements in the domain of continuous noise monitoring and acoustic maps creation. Some results of tests performed using the system prototype are shown. The main focus is put on assessing the efficiency of the acoustic maps created with the discussed system, in comparison to results obtained with traditional methods.
\end{abstract}

Keywords: noise pollution, acoustic noise monitoring, noise propagation model, supercomputing, acoustic maps.

\section{Introduction}

The threat environmental noise poses to human health has become an issue of considerable significance, especially in heavily urbanized areas (KOMPALA, Lipowczan, 2007; Kucharski, 2007; Popescu, Moholea, 2010; Engel, ZAWIESKA, 2004). The problem of noise and vibrations has been undertaken in Poland long time ago. Corresponding research efforts have been dedicated to this subject and many conferences organized as it was recently summarized by Prof. Engel in his paper (ENGEL, 2010). Long exposure to excessive acoustic disturbances is proven to have a negative effect on our physical and mental well-being. The most noticeable result of the following is the constantly decreasing average age of children with hearing loses exceeding $20 \mathrm{~dB}$. Consequently, new methods 
for estimating and reducing noise impact are applied and investigated (KoTUS et al., 2010; CZYZEWsKi et al., 2007; 2008). In some cases application of active methods for noise reduction is justified (PAWELCZYK, 2008).

In order to estimate the threat, the European Parliament and Council issued the legal foundation for undertaking urban noise monitoring in all Member States. The document is referred to as European Directive 2002/49/EC, published on 18/02/2002. Essentially, it obliges European cities with a population exceeding 250,000 to determine the environmental noise level, according to assessment methods used by all of the Member States. The result is presented as a noise map and published in the Internet to ensure that the public is well-informed about environmental noise and its effects. Undertaken studies of noise pollution maps and environmental noise measurements, resulted in raising many projects which aim is to prevent and/or reduce environmental noise wherever its level exceed acceptable value. Furthermore, the Directive acts as a basis for the development of noise reduction solutions.

Based on the above, in recent years, numerous technical means have been implemented in order to assess acoustic climate in European cities. Such solutions are based on two main approaches: noise measurement and prediction. The first approach utilizes a grid of noise measuring stations, working in centralized or decentralized architecture, registering sound pressure level values and associated data. An example of such a system is the grid deployed in Lille, France ${ }^{(1)}$. Alternatively, an acoustic disturbance distribution may be estimated. Such systems are based on noise source and propagation modeling, and were implemented in most large European cities. For instance, the German city of Berlin (Strategic Noise Maps for the city of Berlin, 2008) developed strategic noise maps for road traffic, streetcar and subway, railway, industrial and air traffic noise, calculated according to the $L_{\mathrm{DEN}}$ and $L_{N}$ noise indexes. Similar acoustic atlases are utilized to assess current threats citizens are exposed to, and introduce noise reduction plans in critical locations of the agglomeration (ProBst, 2009).

Both of the highlighted noise monitoring methods have some significant advantages and disadvantages. Live noise measurement provides the most accurate and descriptive results, however it is suitable only for discreet mapping. Implementing a network of monitoring stations sophisticated enough to cover all the key locations in a given city would not be economically justified. Alternatively, predicted noise maps are based on statistical information characterizing modeled noise sources, i.e.: traffic volume, type of road pavement, etc. Hence these data are gathered independently for other purposes, the implementation and running costs of such a system are reduced to the license costs of noise prediction software. However, due to the characteristics of discussed data, generated noise

(1) Noise and weather monitoring combined, last access: 20.06.2008,

http://www.vaisala.com/newsandmedia/vaisalanews/vaisalanews177/noise and weather monitoring combined.pdf 
maps are heavily generalized, sporadically updated and do not consider temporal changes in the acoustic level. Moreover, strategic noise maps need to be generated separately for the types of noise outlined above. Such a chart contains less information than a map calculated employing all the noise sources present in the area. Continuous measurements may contribute to minimizing the measurement uncertainty or to improving the estimators in opposition to short-term fragmentary ones (BATKO, StęPień, 2009).

For comprehensive assessment of environmental noise, there has arisen the need for systemic unification of the two discussed approaches. Such a system has been designed and developed at the Multimedia Systems Department of the Gdansk University of Technology, Poland. Through the grid of monitoring stations, the solution produces noise maps, based on and verified on the basis of live data. The utilized noise prediction model, implemented on a cluster-type computer, allows for fast and accurate updates of generated maps. The featurerich and easily accessible Internet visualization module of the system enables an efficient dissemination of environmental noise threat information, making it available to the general public. The design, implementation and practical results achieved with the developed urban noise monitoring system are presented below.

\section{Continuous noise monitoring}

\subsection{General concept}

The conceived and implemented system presented in this article was designed for the continuous monitoring of acoustic climate in urban areas. The assessment is performed based on online data, acquired through a grid of engineered monitoring stations. The gathered information consists of current noise level values and associated data including: meteorological conditions and registered traffic volume size. Discussed data are utilized to create dynamic noise maps accessible for the common public. The operation is performed by a dedicated noise prediction model, optimized towards working on a computer cluster. This enables the system to generate noise maps in a reasonable time and publish their regular updates in the Internet. Moreover, the discussed charts may present acoustic disturbance originating from different sources (air, road or railway traffic) separately, or as a common distribution, considering negative effects noise has on human health, especially on hearing. The maps would contribute more to the field of public noise pollution awareness than their strategic counterparts defined by the European Directive 2002/49/EC. This is because, being regularly updated and based on measured and accurate data, they present comprehensive information on the acoustic climate in a given area. On the other hand, noise-related data acquired through the discussed measurements, generalized for a defined time interval, may be utilized to generate strategic noise maps of an exceptional accuracy. The reason of the latter is that common strategic noise maps are often 
based on estimated or considerably generalized input data. In addition, predicted maps may be adjusted with real-world noise level measurements through some reverse engineering techniques. This approach allows for generating more precise maps, but also for verifying mathematical source models, utilized in the process. Hence, it seems that the achieved result may contribute significantly to the field of noise modeling and simulation.

\subsection{Dynamic determination of noise source parameters}

The outcomes of the European HARMONOISE or IMAGINE projects provide a base for engineered noise prediction software, as they include the description of methods for modeling road and railway noise ${ }^{(2),(3)}$.

The proposed new concept of obtaining input data of the models is based on acquisition of parameters characterizing the discussed source model through continuous measurements. This significant improvement of data acquisition allows maintaining the accuracy of calculated short-term noise maps. A specialized device, developed by Multimedia Systems Department, is capable to extract necessary information from an image recorded by a camera (DALKA, 2006). Software embedded in the device is suitable for evaluating non-acoustic parameters of any noise source originating from moving objects like trains or cars. The advantage of this device is its ability to set a wide range of angles and distances to the source which makes it easy to place it along a road or railway. The device is capable of recognizing the number of passing vehicles with their classification into desired category groups, determining the average speed of vehicles. For a road noise source these categories can be divided into light, medium and heavy vehicles according to their dimensions.

The gathered data is processed by software, which is using a source model and a sound propagation model in order to obtain noise levels in a given city area. The period of publishing the current map is mainly determined by the time of computing the new sound level distribution over a specified region.

Predicted results are confronted with real-life, measured noise values. This technique is recently referred to as Reverse Engineering (COMEAGAA et al., 2007). Tuning of the model can be performed if its outcomes differ from real noise observed in a specific place.

\subsection{Modeling of the acoustic field distribution}

The work of participants of above mentioned European projects resulted, inter alia, in development of new methods for noise source description. The Harmonoise model was intended to be the one model for European Union state members,

(2) The Harmonoise project, access 23.11.2005, http://www.harmonoise.org

(3) The Imagine project, access 21.10.2006, http://www.imagine-project.org 
and it was also designed to replace all different European models (JONASSON et al., 2004; NotA et al., 2005). Regarding the introduction of new methods, the NMPB-96 model, recommended for the European states which do not have their own standards, was not chosen for implementation. The features of the new model includes more precise calculations (performed in $1 / 3$ octave bands).

In order to define the noise emission of a linear source representing a road, two separate models for vehicle and traffic are distinguished. First, the vehicle model, describes the sound power of a single moving vehicle. The input data is the velocity and the output is the sound power for a specific vehicle type. Each vehicle produces two noise sources at different heights. Second, the traffic model, comprises a statistical description of sound power output of the total traffic flow. Combined noise emission of numerous single vehicles into the sound power per one meter length of the linear source provides output of the traffic model. Although the Harmonoise model assumes a division of road vehicles into 5 categories, only 3 have emission parameters completely defined. All input data have to be provided in 3 categories (for light, medium and heavy vehicles). Corrections can be applied for different pavement types, tires, road topography (slopes), traffic lights and source directivity.

Based on the sound power output for a single moving vehicle $\left(L_{W, m, i}\right)$, and on the average vehicle speed and the traffic flow, the total sound power output $L_{W, m, i}^{\prime}$ of each different source on a unit length road section is defined by Eq. (1):

$$
L_{W, m, i}^{\prime}=L_{W, m, i}+10 \log \left(\frac{Q_{m} v_{0}}{1000 Q_{0} v_{e q, m}}\right),
$$

where $v_{0}$ - the reference vehicle speed $(1 \mathrm{~km} / \mathrm{h}), v_{e q, m}$ - the equivalent vehicle speed for vehicle category $m[\mathrm{~km} / \mathrm{h}], Q_{0}$ - the reference traffic flow $\left(1 \mathrm{~h}^{-1}\right)$, $Q_{m}$ - the traffic flow for vehicle category $m\left[\mathrm{~h}^{-1}\right], i$ - number of the $1 / 3$ octave band, $i=1,2 \ldots 31, m \in\{1,2,3\}$.

The total sound power output of a unit length road section is obtained by summation over the different vehicle categories.

The sound level in each receiver is obtained using the propagation model allowing for calculating the total sound level in a defined set of points called receivers. The Harmonoise method describes the attenuation between each point source and an arbitrary receiver point (BARELDS, NotA, 2002; EMBLETON, 1996). The general formula representing the sound level in a certain point, including factors that affect propagation, is calculated according to Eq. (2) (ENGEL, 2001):

$$
L_{p}=L_{w}-20 \log (r)-11+D-A_{\mathrm{abs}}-A_{E},
$$

where $L_{p}$ - sound pressure level $[\mathrm{dB}]$ (ref. to $2 \cdot 10^{-5} \mathrm{~N} \cdot \mathrm{m}^{-2}$ ), $L_{w}$ - source sound power level $[\mathrm{dB}]$ (ref. to $\left.10^{-12} \mathrm{~W}\right), r$ - distance from source to receiver $[\mathrm{m}]$, $D$ - directivity index $[\mathrm{dB}], A_{\text {abs }}$ - atmospheric absorption $[\mathrm{dB}], A_{E}-$ an excess attenuation $[\mathrm{dB}]$. 
The total excess attenuation $A_{E}$ is a combination of all propagation factors, mainly meteorological conditions, influence of ground, vegetation. The described model uses a concept of the sound propagation paths representing the schematic, straight-line tracks of the sound waves between source and receiver points, where point sources are obtained by segmentation of the linear source.

Finally, the model yields the total, short-term, $A$-weighted noise level at the receiver point which is determined by summing up all contributing point sources over the propagation paths.

\section{The system}

This section presents a general overview of the created system. Apart from describing the main components of the system and on outlining their functionality, the main focus is put on presenting the internal structure of main system modules and outlining techniques used to implement them.

\subsection{System architecture}

The system architecture is shown in Fig. 1. The system was designed to conform to international standards, and to ensure robustness, scalability and open architecture demands (HARMOn, AndERson, 2003).

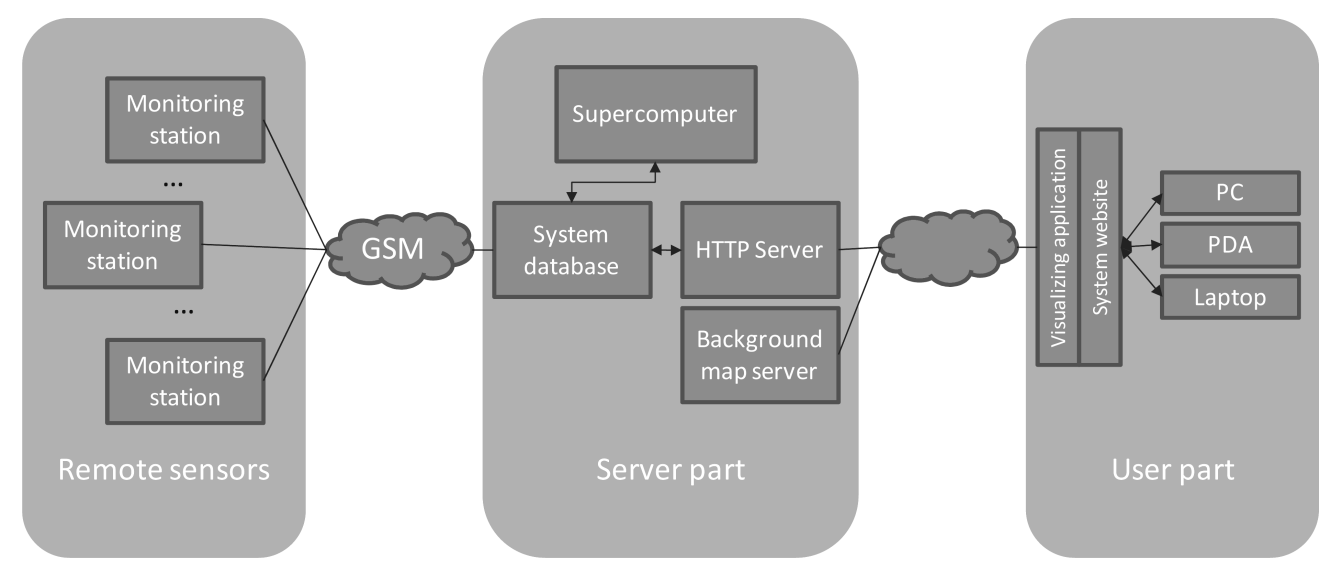

Fig. 1. System architecture.

The engineered system is based on a grid of noise monitoring stations. Those devices are based on a miniature, nevertheless efficient, industrial PC and set of sensors to a acquire sound pressure level, together with associated traffic parameters and meteorological data. Utilized sound acquisition devices are Class-1, sound meters implementing the authors' concept (CZYZEWSKI et al., 2005). Noise monitoring stations are also equipped with a GPS receiver and means of wire- 
less communication (UMTS/EDGE or GPRS modems). Data acquired by the discussed grid is transferred to the system database. The database stores and provides information being visualized by the system: predicted or real noise level values, registered weather and traffic conditions. In addition, configuration information for all other system components is stored there as well. The HTTP server serves as a data provider for the visualizing application. Moreover, it manages connection status, user validation and data transfer. As seen from the user perspective, it is the only visible part of the server-side segment. The background map server provides the visualizing application with a geographical context. The most important part of the discussed architecture is the noise prediction model software which contains algorithms for generating the noise map, implemented on the cluster-type supercomputer. The system as above constitutes an efficient data acquiring and computing architecture for determining noise distribution on a given urban area, with regard to selected noise indicator. The output data is organized as geo-referenced, uniform raster of values, ready to be delivered to the visualizing application. The system website provides a' middleware between the server-side and the user segment of the system. The website houses the visualizing application and presents textual information, in a response to user queries. The visualizing application is implemented as a standard Internet browser plugin, differing in its functionality in the case of PDAs and desktop computers. It performs all operations concerning aggregating, processing, displaying and animating geographically-related data.

\subsection{Data acquisition}

The prediction model utilizes static and dynamically acquired data to noise map generating. The first group consists of spatial data in the form of a numeric map. The second group is composed of information, gathered by the grid of monitoring stations. The type of data is determined by the acquisition capabilities of a given station and mostly include traffic parameters plus weather and air quality information.

The discussed static data define all architectural barriers a propagating sound wave may encounter, when passing through or is reflected from those barriers. Significant information for the environmental acoustics is carried out with the ground elevation model. This layer, supplied usually by municipal authorities, is used to define terrain topographic features. Moreover, the discussed layer also defines the ground type in a given location. All of these features contribute significantly to the resultant noise pressure level calculated for a given location. Other static data define architectural obstacles for the propagating noise. The most important one describes buildings (type, material of facades, height, etc.) and greenery. Knowledge of the location of acoustic sources is vital for the noise source modeling algorithms. It is carried with numeric maps of roads and railways. 
Each monitoring station is modularly designed to support various acquisition devices, i.e.: noise meter, weather station, traffic camera and air quality sensor. Noise meters, preinstalled in all active monitoring stations, are Class-1 products designed and built at the Multimedia Systems Department (CZYZEWski et al., 2005). They consist of a noise measuring microphone and USB acquisition module. The device is capable of acquiring a sound pressure level with dynamics of $120 \mathrm{~dB}$. The discussed meter is fully configurable in terms of acquisition time, applied filter correction curve (A, C) used in acoustics, and sensitivity. Information of the traffic volume and characteristics is acquired through a high resolution IP camera, shown in Fig. 2d. Weather conditions are known to contribute to the acoustic climate of a given area. Therefore the monitoring stations are equipped with meteorological sub-stations. Those devices measure typical parameters affecting noise propagation, such as: temperature, humidity, atmospheric pressure, wind velocity and direction, etc. An optionally installed air quality sensor acquires information about the concentration of benzene, dusts and all other harmful airborne factors. Data acquired through the discussed peripherals are stored in the local memory of the station and uploaded to the system database in regular intervals. The working status and measuring settings of the station may be viewed and adjusted remotely by the system administrator. Similarly, in the case of a peripheral device failure, a diagnostic panel may be accessed.
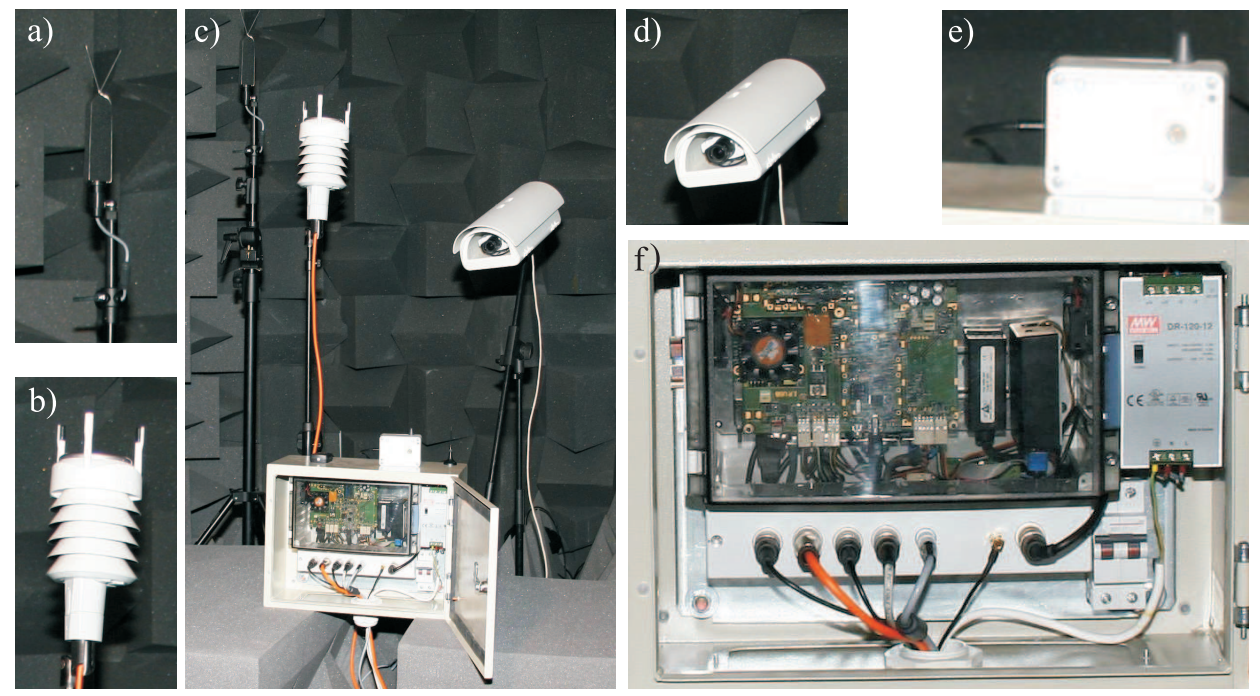

Fig. 2. Noise monitoring station: a) weatherproof measuring microphone, b) integrated weather station, c) complete monitoring station with all sensors, d) digital camera, e) air sensor, f) central monitoring unit (for data processing \& remote control).

Overall, the designed and implemented solution represents a technically advanced noise monitoring station. Due to its mobility, modularity and compact casing, this device may be used to monitor acoustic climate in closed and opened 
spaces. Based on open source software components and utilizing a low power consumption central unit, the discussed station is characterized by low introduction and running costs. Moreover, acquiring comprehensive information about an acoustic climate, the grid of the proposed devices may provide valuable data for the purposes of long and short time acoustic analysis. The sample station images are presented in Fig. 2c.

\subsection{Noise propagation model implementation}

The aim of dynamic noise mapping is to depict the spatial distribution of the sound level in a certain period of time. Employing a typical personal computer to complete the task of calculation of the map would result in taking a huge amount of time. A real risk exists that it may become longer than the period in which the map should be updated. Consequently, methods for acceleration of computation should be used. One of them is the parallelization of the algorithms and application of a multiprocessor computer. Suitable hardware provides the computer cluster installed at the T.A.S.K. Academic Computer Center ${ }^{(4)}$ of Gdansk University of Technology. The theoretical computational power of the cluster employing 1344 quad core processors reaches 50 TFLOPS. In comparison to a typical modern personal computer with $2 \mathrm{GHz}$ speed of singular GFLOPS, it performs calculations several thousand times faster. The computational capacity makes it one of the fastest computers in Poland and in the surrounding European region.

Implementation details of the previous version of software called Noise Prediction Model (NPM) employing the Harmonoise methods were described in our earlier paper (Szczodrak, Czyzewski, 2008). Nevertheless, the main issues concerning implementation are recalled here. The Harmonoise model completely separates sound emission and sound propagation. Such a convenient division allowed for implementing the easier road source model first and supplementing the software later by using railway noise source model. The road source model and the propagation model were implemented using some free programming tools and open-source libraries. The main engine of the propagation model employs the acoustic ray tracing method (Kulowski, 1991; Li et al., 1998). A set of rays is sent from each receiver point. The checking of the collision of the ray with buildings or roads is performed. Every ray and object situated in its range is examined in order to find point source or obstacle reflection during each iteration. Geometrical cross-sections called propagation paths, representing paths along which acoustical energy is transmitted from the source point to the receiver point, are determined.

The propagation path, as shown in Fig. 3, is determined in a 2-dimensional space, in the horizontal projection. The Point-to-Point programming library, developed initially by the Harmonoise project, is aiding calculation of attenuation

(4) TASK Academic Computer Center, http://www.task.gda.pl 


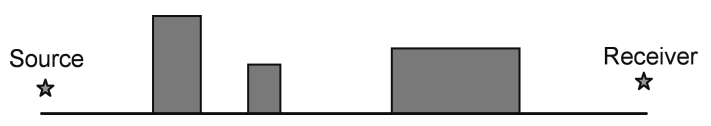

Fig. 3. Sample cross-section of the propagation path.

between a single source point and the receiver point. The total noise level in a given point is attained by summing up all contributions from point sources.

The software is designed to operate on a computer cluster. The considerable advantage of the modeling method which separates the source and propagation parts is that the sound level in each given point can be obtained independently. The software implementation is using the MPI ${ }^{(5)}$ programming standard. The designed algorithm architecture uses a master - slave approach. This significantly increases the overall Noise Prediction Model performance, as all available computer cluster cores are equally charged in this case. The diagram illustrating the application of the computer cluster is shown in Fig. 4.

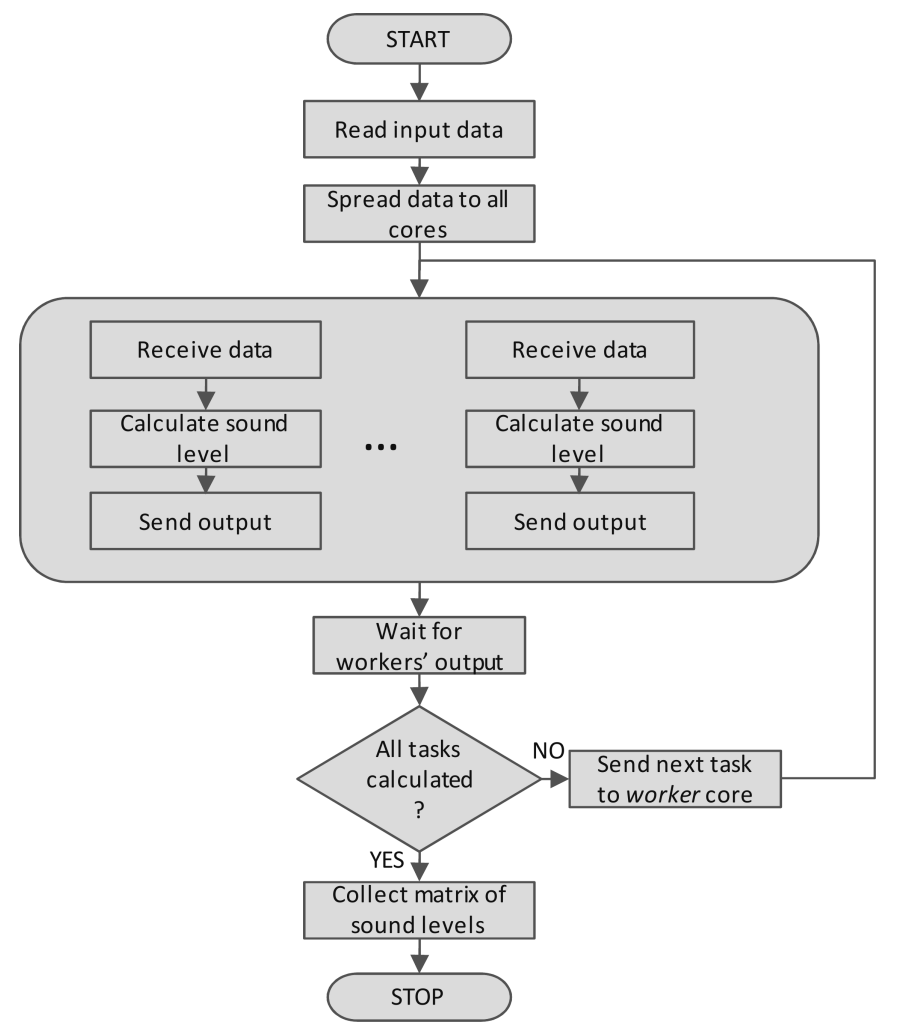

Fig. 4. Algorithm of noise map calculation by computer cluster.

(5) Message Passing Interface - is a specification of an application programming interface allowing many computers to communicate with one another. It is used in computer clusters and supercomputers. 
The main input data for the Noise Prediction Model provides a grid of points in which the noise level should be calculated. The master core manages data flow and communication within processors. It controls and distributes the computational tasks to the remaining cores. The computational task is understood here as obtaining the sound level in a given point. The algorithm stops when all tasks were processed. Output data represent noise distribution in a specified region and appear in the form of an XML file or raster image.

\subsection{Data visualization}

The visualization of dynamic phenomena in the Internet is a demanding task. This is due to the fact that a conventional Web browser is not able to visualize fully dynamic geographical data. The most common way of presenting such a kind of information is as a set of static images, combined with textual information. This kind of a presentation is insufficient for the purposes of the discussed system. Whereas hitherto available GIS solutions include well-developed Internet modules dedicated to visualize static maps there are virtually none of them capable of producing dynamic noise maps. Therefore, a new concept-based tool to visualize dynamic noise maps in the Internet was designed and implemented following some ideas presented in the literature (FURLANELLO et al., 2002).

The discussed application was implemented as a standard Internet browser plug-in. Therefore it fulfills the 2002/49/EC Directive with regards of providing common, public access to the generated maps. The sample interface of the application is shown in Fig. 5. Currently, its displays are edited in the Polish only.

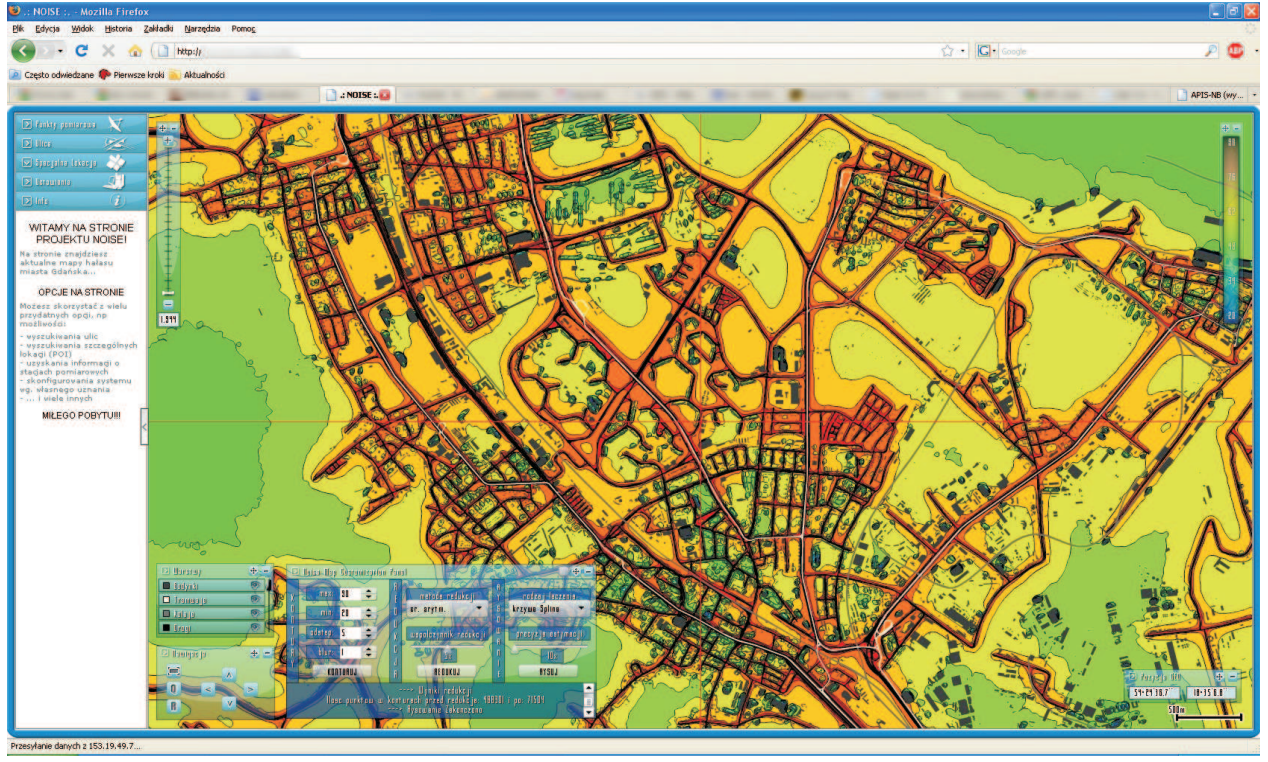

Fig. 5. Sample application interface. 
The main feature of the application is its ability to generate noise maps automatically. Therefore, information required to create such a map (array of noise level values and georeference data) is sent to the plug-in in a raw, numeric format. After a thorough consideration, vector graphics have been chosen as a primary medium for presenting the system output. This constitutes a novel approach, when compared to the commonly used solutions presenting noise maps as raster images. Presenting acoustic charts in the proposed way is economically justified, in terms of decreasing system running costs and lowering demands for server-side resources. Moreover, the discussed approach enables the user to assume control over the visual output of the system, and thus to contribute to the map generation process on an unmatched level of flexibility. This is done through manipulating certain parameters including noise map: color scheme, number of layers, applied curve approximation technique, etc. In order to transform input data into the graphical form, the application has been implemented with algorithms for contour extraction, labeling and optimization. As a result, a set of vector shapes visualizing environmental noise distribution is created. In order to add geographic context to the presentation, the discussed map is geographically positioned on the underlying map of an agglomeration. Dynamic changes or regularities in acoustic disturbance distribution may be highlighted additionally by animation. Apart from the discussed features, the application is capable of visualizing other media, such as temperature or atmospheric pressure, or discrete objects (i.e. POIs).

The simplified outline of the algorithm for the dynamic noise maps generation is shown in Fig. 6. It consists of three main sections, dedicated for contour

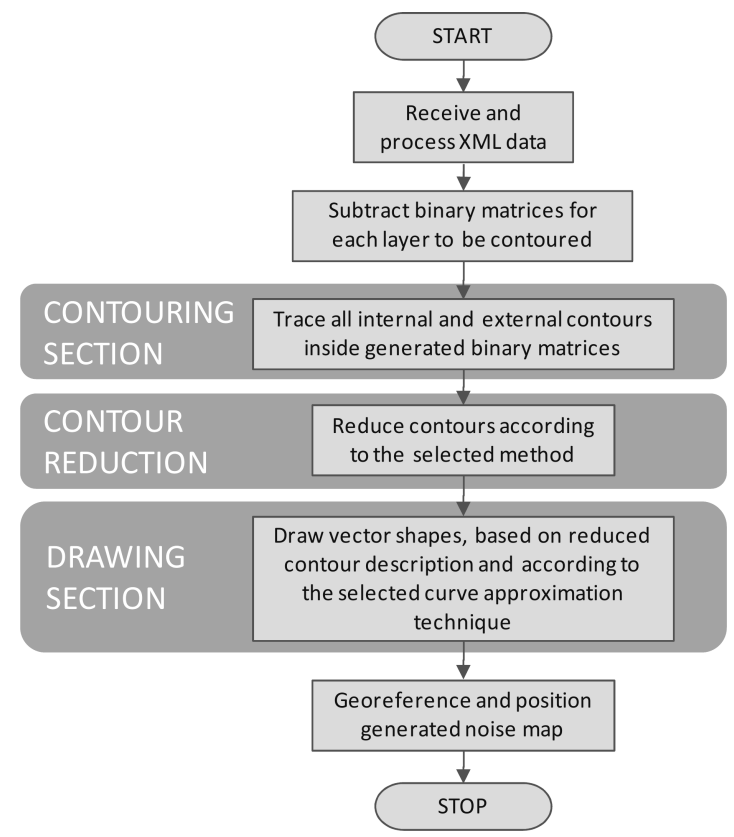

Fig. 6. Dynamic noise maps generation algorithm. 
extraction, reduction and drawing. The contour extraction subroutine operates on preprocessed input data - a georeferenced matrix of calculated SPL values. Generally, it is an adaptation of the solution proposed by CHANG et al. (2004). However, the descriptions of extracted contours are highly redundant and need to be reduced before drawing. This is performed through various curve approximation techniques, i.e., the Lagrangian Method. The extracted and reduced shapes are drawn with the user-selected technique, i.e., Bezier Splines. The choice is determined by the target scale of the map and desired application performance. The sample output of the discussed algorithm, in comparison with the raster noise map of the same scale, is presented in Fig. 7.

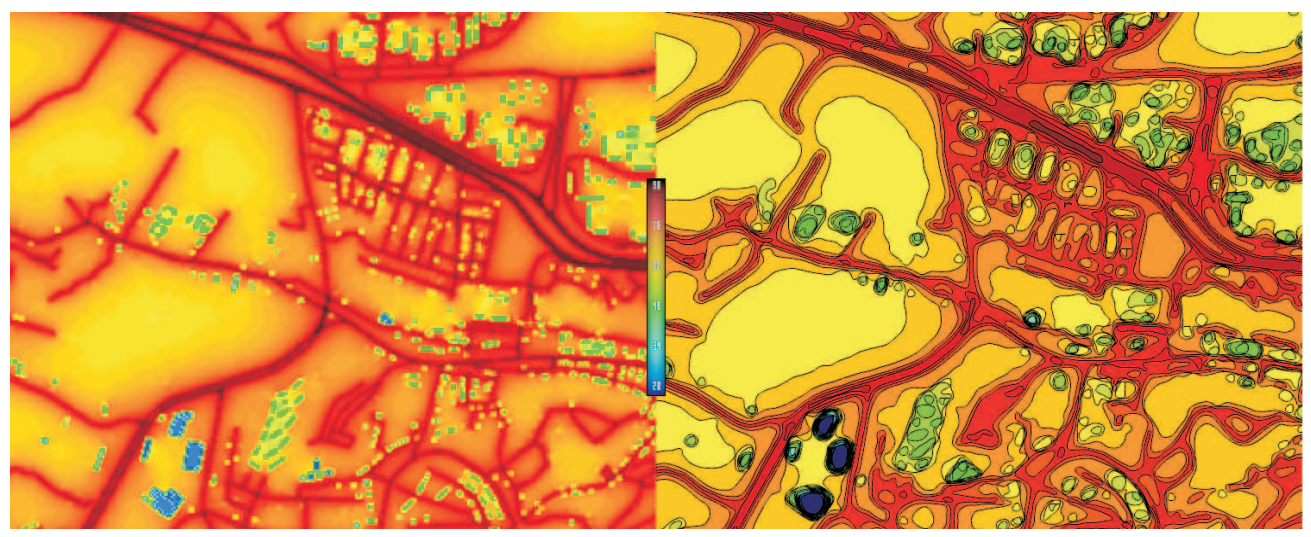

Fig. 7. Comparison of raster and vector noise maps (the latter obtained as an algorithm output).

\section{Examining system performance}

This section describes performed experiments. The initial subsections below focus on comparing the engineered hardware and the implemented Noise Prediction Model output to commercial software application results and to measurement results obtained with reference measuring equipment. The subsequent 2 subsections compare the performance of the noise map creation for a large area. The sound levels which are described in this paragraph were $A$-weighted and referenced to the pressure level of $2 \cdot 10^{-5} \mathrm{~Pa}$ (International Standard, 1990).

\subsection{Locations of measurements}

The measurements were conducted in 3 different locations, denoted in the following text by letters: A, B, C. In the first location (A) both short- and longterm sound level measurements were carried out, in the two next places only one type of measurements were performed, the short-term (B) and the long-term (C), respectively. The situation plan and microphones setup for measurement sites is shown in Fig. 8. 

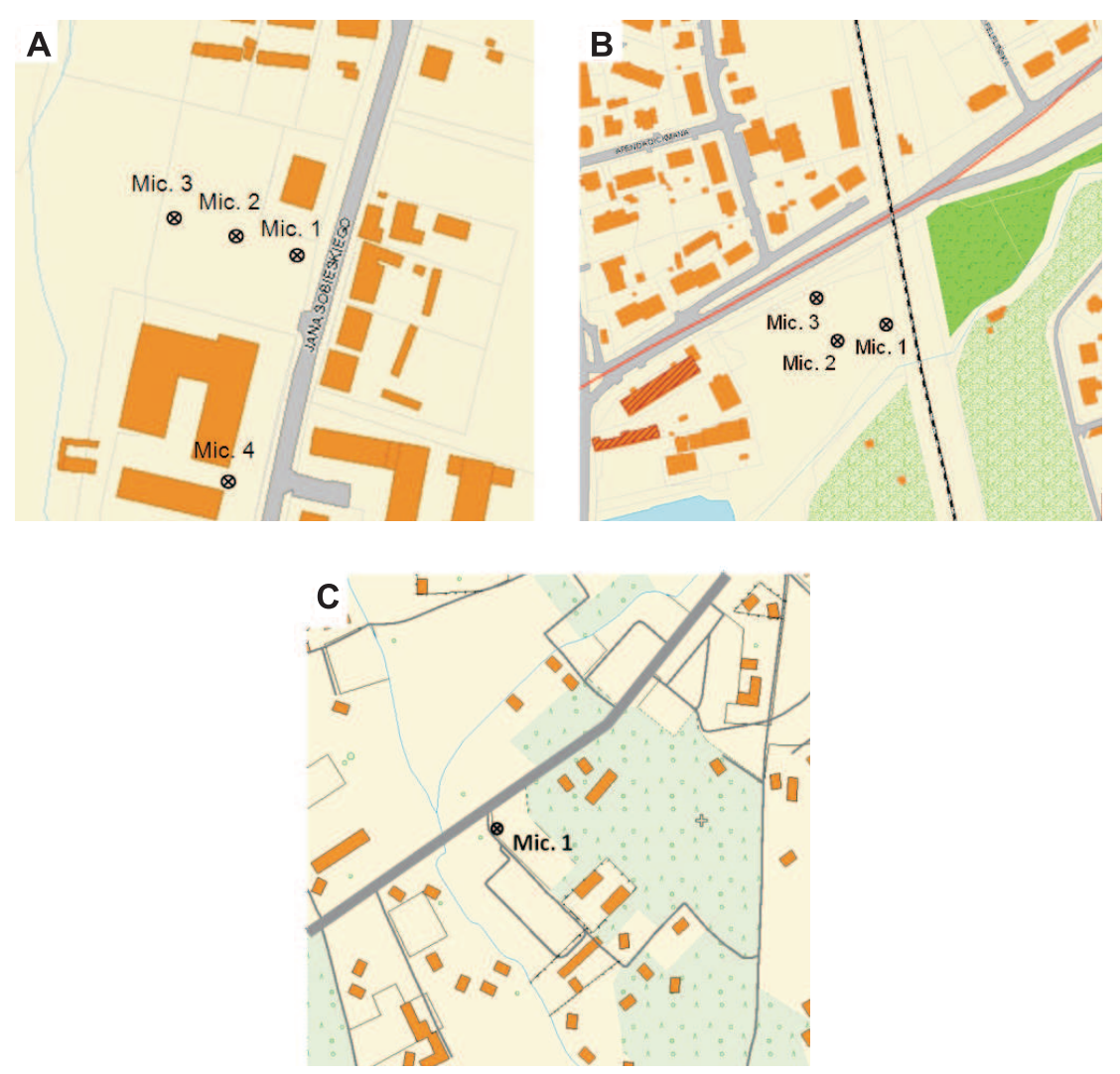

Fig. 8. Details of microphone placement, locations 'A', 'B' and 'C', scale not maintained.

Location ' $\mathrm{A}$ ' is situated in the city near the road which is practically the only source of noise in the neighborhood. Although traffic is high on this road section, the average speed is low because it extends between two crossings. The road has one lane in each direction. The ground surface between the source and microphones is covered by grass. The short-term measurements in this location were aimed at validating the propagation model.

In location ' $\mathrm{B}$ ' more diversified noise sources were present including a road, a streetcar and a railroad. The railroad overpass crosses the road which is common with the tram tracks. The railroad has 4 tracks, the road has 2 has lanes in each direction, and the tram has 2 tracks. The train traffic is rather high as suburban trains are running at a 15 minute frequency. The ground is covered by grass. Only short-term measurements were done in this place.

Location ' $\mathrm{C}$ ' is in a village outside of Gdansk near a straight section of a national road. Most vehicles are typically traveling at a high speed (approx. $80 \mathrm{~km} / \mathrm{h}$ ) on this road. The ground between the microphone and the road is covered by grass. In this place only long-term measurements were carried out. 


\subsection{Methodology of measurements}

In case of the short-time measurements, two different systems were used at the same time: the engineered monitoring stations and the Brüel\&Kjær PULSE analyzer. The latter was used as the reference system, so that all measurements and simulations results were compared to it.

The measurement microphones (No. 1 to No. 3 in locations 'A' and 'B') were placed at 4 meters above the ground surface. Moreover, source parameters (road traffic volume, number and type of trains and streetcars) and meteorological data were also acquired. The monitoring stations used all-weather microphones produced by the Polish company Sonopan (type WK21WP). The PULSE system cassette type 3560C and microphones type 4189A21 were employed as the second measuring system. Noise samples were recorded by the Time Data Recorder software (v. 12) and analyzed offline using the Pulse LabShop (v. 12) software. All microphones diameters were equal to $1 / 2$ ". Before the measurement was started, all systems were time-synchronized and calibrated with a B\&K 4231 acoustic calibrator. Both systems were powered by batteries. The time duration of measurements was equal to 1 hour. One of the monitoring stations is depicted in Fig. 9 during the work (without outer enclosure).

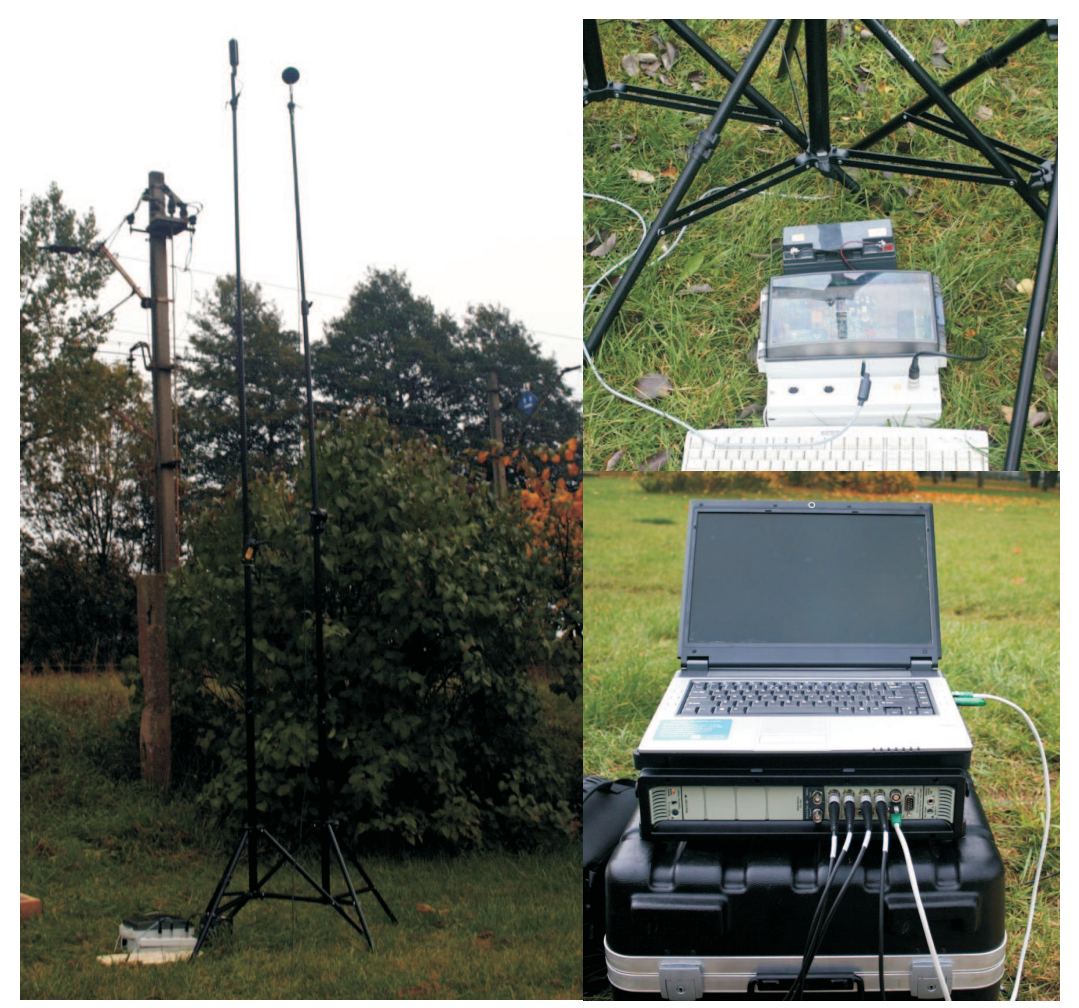

Fig. 9. Engineered monitoring station and PULSE system employed to short-term outdoor measurements. 
The automatic noise monitoring stations were permanently installed in locations ' $\mathrm{A}$ ' and ' $\mathrm{C}$ ' and employed to long-term measurements. Each station was additionally equipped with a digital camera for analyzing the traffic flow. The monitoring stations also offer the function of recording noise events. These stations have been gathering data continuously for 7 days. The traffic characteristics of both analyzed roads were very similar and contained 1200 vehicles per hour during peak time. Instantaneous noise level and traffic statistics were recorded in one minute intervals. All noise measurements results were averaged for 1 hour in order to match the input and the output of the numerical road noise source model (NoTA et al., 2005; NMPB-Routes-96, 1997). The long-term measurements microphone in location 'A' (Mic. 4) was installed $20 \mathrm{~m}$ from the road axis, $6.5 \mathrm{~m}$ above the ground. In location ' $\mathrm{C}$ ' the microphone is $50 \mathrm{~m}$ from the road axis on $5 \mathrm{~m}$ above ground. The photograph of the monitoring station fixed on the aerial mast in location ' $\mathrm{C}$ ' is presented in Fig. 10.

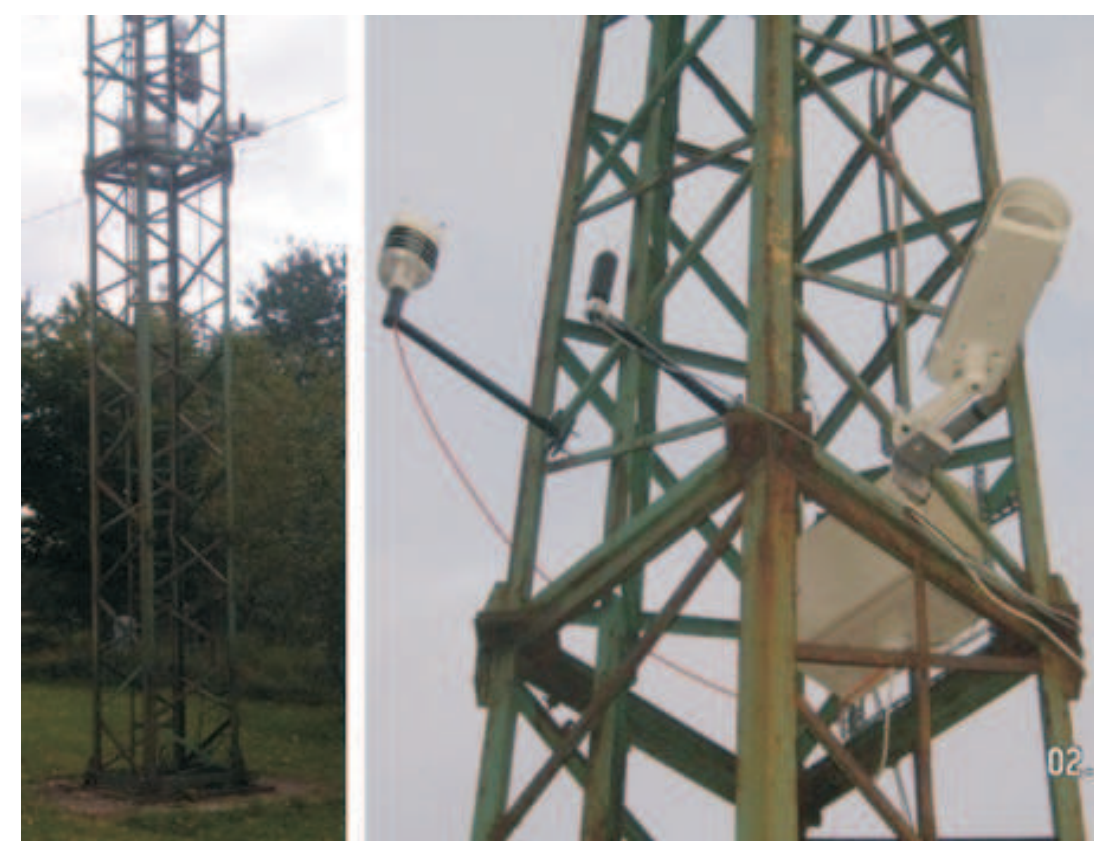

Fig. 10. Noise station installed for long term measurements in location 'C'.

\subsection{Measurements and simulations results comparison}

\subsubsection{Short-term measurements}

During the short-term measurements conducted in location ' $A$ ' the observed number of noise sources were: 1015 passenger cars, 38 trucks and 6 motorcycles. In location ' $\mathrm{C}$ ' there passed 1124 passenger cars, 18 trucks and 9 motorcycles, 
14 suburban trains, 4 passenger trains, 1 intercity train, 3 freight trains, 23 streetcars. In location ' $\mathrm{A}$ ' differences were observed between CadnaA and our Noise Prediction Model of $1.3 \mathrm{~dB}$ in mic. $1,0.8 \mathrm{~dB}$ in mic. 2 and $-0.8 \mathrm{~dB}$ in mic. 3 . The detailed description of results of the short-term measurement employed the Brüel\&Kjær PULSE system and engineered monitoring stations are presented in our previous paper (SzCZODRAK et al., 2008). The comparison results of PULSE measurements to our monitoring station (KSM) and to simulations obtained with CadnaA (CNA) for the location 'B' are included in Table 1.

Table 1. Measurements and simulation results for location ' $\mathrm{B}$ '.

\begin{tabular}{|c|c|c|c|c|c|c|}
\hline \multirow{2}{*}{ Mic. no. } & \multicolumn{3}{|c|}{$L_{A, E q}[\mathrm{~dB}]$} & \multicolumn{3}{c|}{ Difference [dB] } \\
\cline { 2 - 7 } & PULSE & KSM & CNA & KSM - PULSE & CNA - PULSE & CNA - KSM \\
\hline 1 & 70.3 & 70.4 & 66.7 & 0.1 & -3.6 & -3.7 \\
\hline 2 & 65.4 & 65.8 & 65.9 & 0.4 & 0.5 & 0.1 \\
\hline 3 & 67.8 & 68.2 & 67.3 & 0.4 & -0.5 & -0.9 \\
\hline
\end{tabular}

In location ' $\mathrm{B}$ ' the comparison was made only with the use of the commercial program, because the rail noise source was not yet implemented in the Noise Prediction Model software. For rail and streetcar noise the Dutch model was adopted (Reken en Meetvoorschrift Railverkeerslawaai, 1996). The latter is recommended as the railway noise model for countries which do not have their own model. The investigations of this model in the case of Polish trains have been made at the Poznan University of Technology (Golebiewski, Makarewicz, 2009).

The right part of Fig. 11 presents the noise map for location 'B' obtained using CadnaA software. In this case the measured noise level in point 1 (closest to the railway) was significantly higher than the one computed by the software.
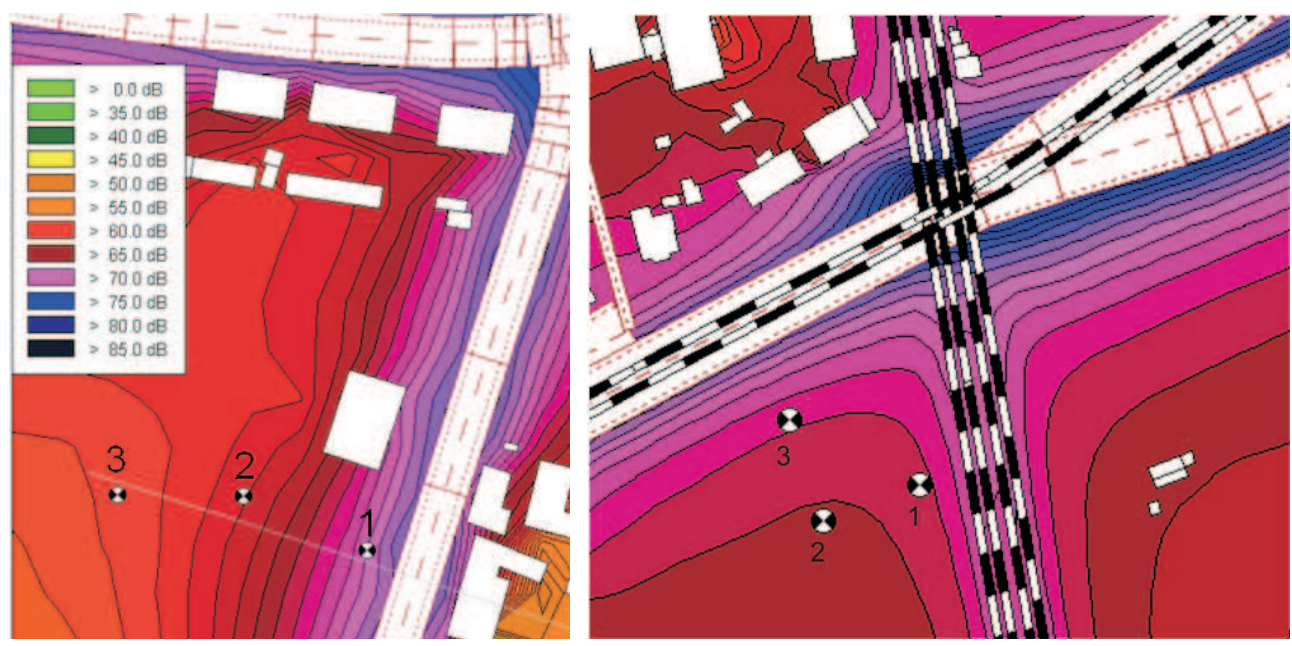

Fig. 11. Short-term noise maps for locations 'A' (left) and 'B' (right). 
The rail noise model brought underestimated results. The PULSE system and the monitoring stations measurement results were nearly identical.

\subsubsection{Long-term measurements}

Long-term noise measurements results in locations ' $\mathrm{A}$ ' and ' $\mathrm{C}$ ' were compared to the values computed with software. The measured road traffic noise source parameters provided the input data for the noise calculation software. The averaged 1-hour traffic density and speed data gathered from long-term measurements were introduced to the model. The results of comparison to the NMPB-96 model and to the Harmonoise model for location 'A' are presented in Fig. 12 and for location 'C' in Fig. 13.
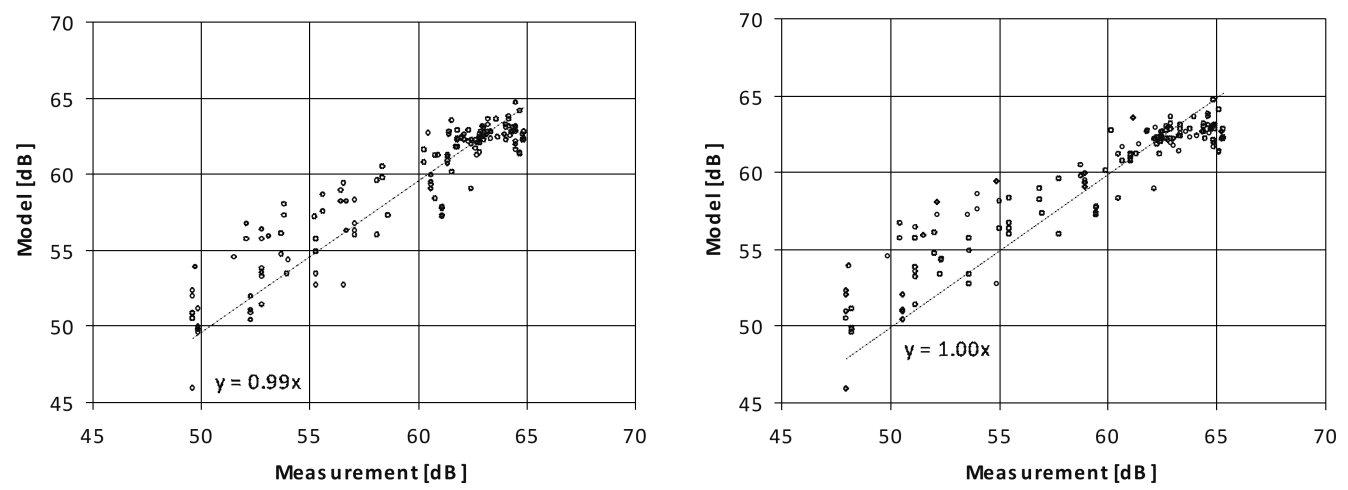

Fig. 12. Comparison of the NMPB-96 model (left) and the Harmonoise model (right) in location ' $\mathrm{A}$ '.
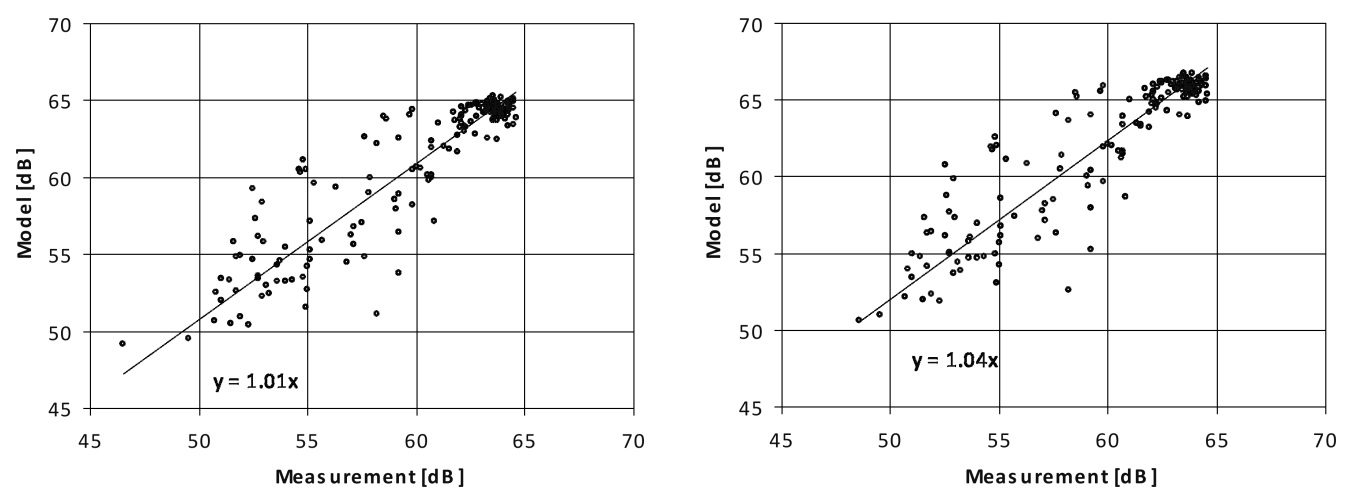

Fig. 13. Comparison in location 'C' of the NMPB-96 model (left) and the Harmonoise model (right).

The measured noise level is similar to the one obtained by the model in a prevailing number of points. In the case of both ' $\mathrm{A}$ ' and ' $\mathrm{C}$ ' localizations the regression line is nearly ideal. The achieved correlation coefficients equaled 0.84 
for the Harmonoise, and 0.82 for the NMPB model in location 'A'. Consequently, values 0.82 and 0.83 in location ' $\mathrm{B}$ ' were obtained. Sometimes considerable differences between measured and computed sound levels may occur. Increased values of the real sound level can be caused by e.g. vehicles using a car horn or "tuned" motorcycles. Moreover, various loud ambient sounds (e.g., construction works) cause that instantaneous noise levels differ from those which should occur for a given value of traffic volume.

\subsection{Large area noise map comparison setup}

Calculations of the noise map for a large area were performed in order to compare the implemented model to commercial software. The parameters of the considered area were as follows:

- dimensions $3,200 \times 3,200 \mathrm{~m}$,

- raster $8 \times 8 \mathrm{~m}$,

- 160,801 points to calculate sound level.

The following main parameters of computations were set as follows: reflections of 1st order, search ray 2,000 meters, distance between following rays 2 degrees.

The input data consisted of:

- Geometrical description of roads;

- Geometrical description of buildings;

- Traffic volume (fixed for all roads: 3000 light vehicles/h, 100 medium heavy vehicles/h and 50 heavy vehicles/h) and vehicle speed $(50 \mathrm{~km} / \mathrm{h}$ for all categories);

- Buildings sound reflection coefficient (fixed value 0.8 for all buildings).

All other parameters in both programs were set to default values, i.e. pavement type of stone mastics asphalt, uninterrupted traffic flow, zero slopes on routes. The ground type for entire area was set as hard (representing asphalt or concrete), because detailed data were still unavailable. Some inconsistencies in the ground type setup were noticed. The commercial software strictly implements the norm ISO 9613 ground attenuation model, where $G=0$ coefficient means hard ground, and the Noise Prediction Model uses a more detailed flow resistivity parameter which value was set to $20000 \mathrm{kN} \cdot \mathrm{s} \cdot \mathrm{m}^{-4}$ (International Standard, 1990). Calculated maps show a sound level $L_{A, E q}$ averaged for 1 hour.

\subsection{Large area noise map comparison results}

The graphical representations of the results obtained with the commercial software and with the Noise Prediction Model are shown in Fig. 14 and Fig. 15. For a better visibility, maps in these illustrations depict a quarter of the specified area $(800 \times 800 \mathrm{~m})$. Figure 16 and Fig. 17 present respectively the difference map showing the commercial software results subtracted from Noise Prediction Model results, and also the result of the opposite comparison. 


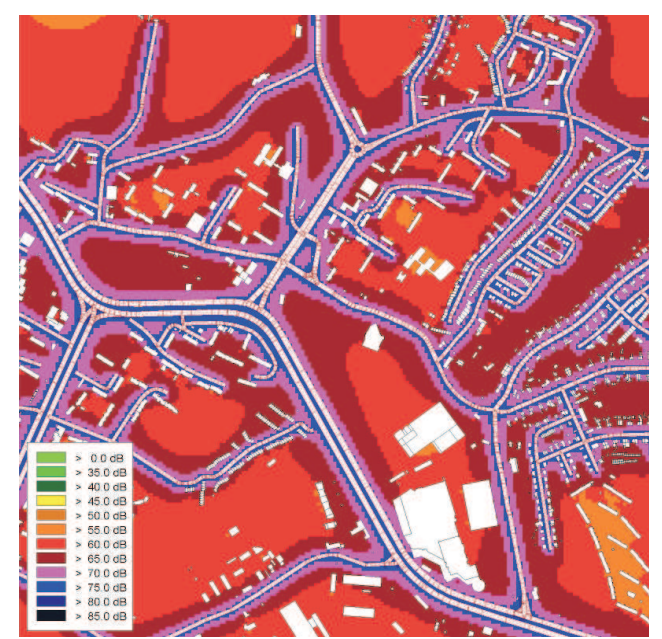

Fig. 14. Noise map, CNA.

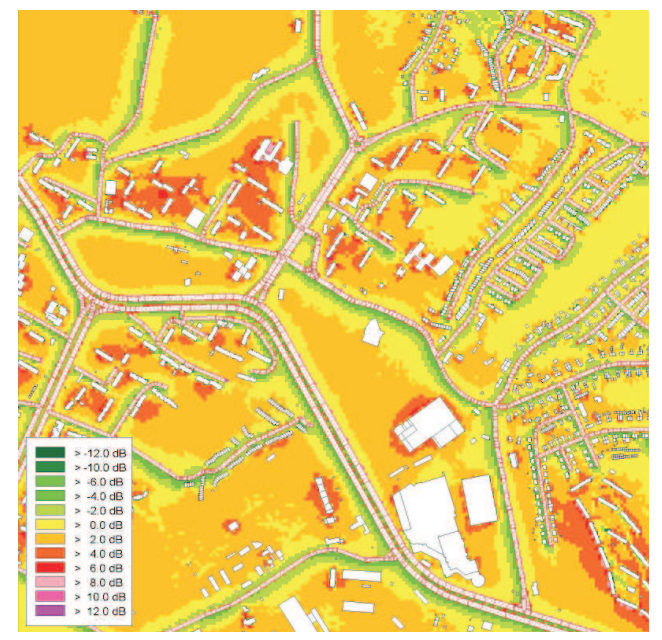

Fig. 16. Difference map NPM-CNA.

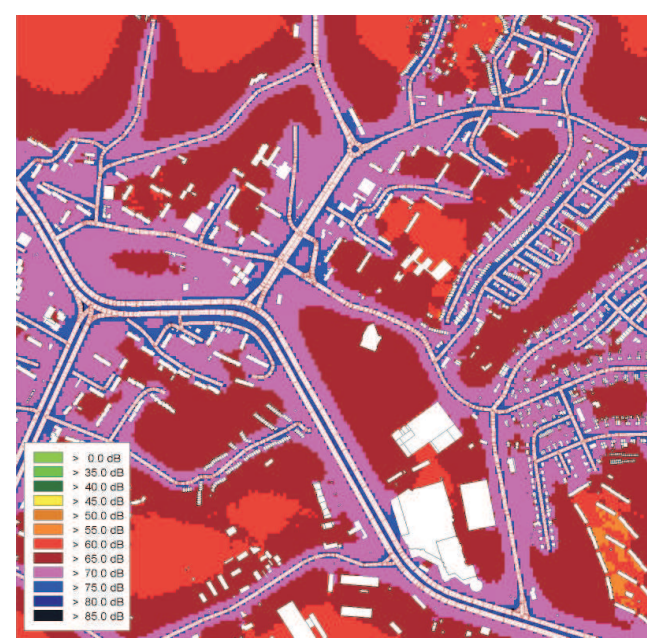

Fig. 15. Noise map, NPM.

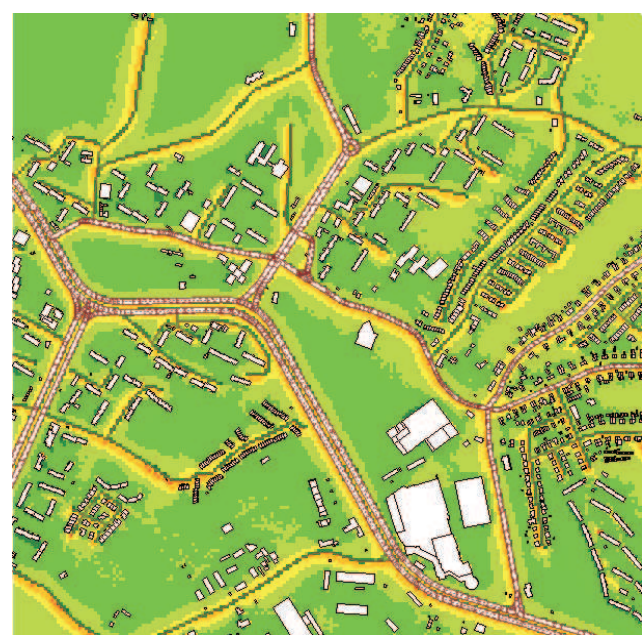

Fig. 17. Difference map CNA-NPM.

The values of noise level in each point of the grid were subtracted in order to obtain the exact differences. Graphical comparison would be insufficient because the sound levels on both maps were quantized to 5 -dB ranges and then colored.

In comparison to our model, the commercial software estimates a higher noise level near the borders of the roads and smaller when the distance from the source is larger. The biggest differences between models $(4-8 \mathrm{~dB})$ are noticed near high buildings and their aggregations surroundings. The propagation part of the Noise Prediction Model in this test case underestimated the attenuation of sound. Regions not urbanized indicate $2-4 \mathrm{~dB}$ larger noise levels calculated by the Noise Prediction Model. The propagation part of NPM have to be tuned up by decreas- 
ing the flow resistance parameter of the first order of magnitude corresponding to a compacted dense ground.

The speed of computation was also investigated for large maps (each for a quarter of area $3200 \times 3200 \mathrm{~m}$ ). The set of experiments was performed. The software was run several times for each map. The number of employed cores was increasing. One of the measures of efficiency of the parallel program adopted is the speedup coefficient defined in Eq. (3),

$$
S(n, p)=\frac{T(n, 1)}{T(n, p)}
$$

where $n$ - task size, $p$ - number of cores, $T(n, p)$ - time of execution on $p$ cores.

As the noise level in each point can be calculated independently, the speedup coefficient should be theoretically close to the number of cores engaged in each experiment. The described investigations aimed at determining the dependence of computation time and number of engaged processor cores. Obtained values of the speedup coefficient are shown in Table 2 .

Table 2. Computation speedup coefficient for maps 1 to 4 .

\begin{tabular}{|c|c|c|c|c|r|r|r|r|}
\hline \multirow{2}{*}{ Number of cores } & \multicolumn{4}{|c|}{ Computation time [s] } & \multicolumn{4}{c|}{$S(n, p)$} \\
\cline { 2 - 9 } & Map 1 & Map 2 & Map 3 & Map 4 & Map 1 & Map 2 & Map 3 & Map 4 \\
\hline 64 & 8395 & 7759 & 8078 & 8714 & 63.8 & 63.5 & 63.6 & 63.9 \\
\hline 128 & 4199 & 3852 & 4011 & 4358 & 127.5 & 127.9 & 128.0 & 127.7 \\
\hline 256 & 2095 & 1928 & 2007 & 2174 & 255.5 & 255.7 & 255.8 & 256.0 \\
\hline 512 & 1048 & 963 & 1003 & 1088 & 510.8 & 512.0 & 511.7 & 511.8 \\
\hline 1024 & 523 & 482 & 502 & 544 & 1024.0 & 1022.4 & 1022.8 & 1023.9 \\
\hline
\end{tabular}

The dividend $T(n, 1)$ in Eq. (3) have an estimated value, which represents the worst case, according to Eq. (4). The simplification is made because the computation time for 1 core would be very long.

$$
T(n, 1)=\min \left(t_{1,64}, t_{1,128}, t_{1,256}, t_{1,512}, t_{1,1024}\right),
$$

where $t_{1, p}$ is the computation time on $p$ cores multiplied by $p$.

The outcomes show linear dependence of the computation time and the number of cores. Each doubling of computational power results in halving the time of computation. Moreover, the earlier investigations show that a complete noise map for the city of Gdansk can be prepared in less than 48 hours (SzCZODRAK, Czyzewski, 2009).

\section{Conclusions}

As a result of the described project, the online acoustic noise monitoring system was implemented, installed and tested in the area of Gdansk, Sopot and Gdynia (the Polish Tricity). The implementation of the software for the 
acoustic climate assessment, consisting of the Source Model and the Propagation Model, was performed. It was optimized towards working on the computer cluster, and thus accelerating the noise maps generation process. Through a unique approach to the map generation process, the dynamic noise maps may be presented to the public in a convenient and attractive manner. Furthermore, the system requirements for server-side hardware resources and network bandwidth were significantly decreased. The utilization of real measured data allows for achieving unprecedented accuracy of short-term and long-term sound level distribution visualization. The developed software was tested with pre-verified data which enabled a validation of the noise propagation model employed. It was important to note that the implemented source model worked properly. The conducted long-term pilot measurements and comparative analyzes allow for stating that a high compatibility between measurement and model outcomes has been achieved. Moreover, the monitoring stations and the PULSE system returned compatible results. Experimental results reveal the importance of the accurate dynamic noise maps creation process. The achievement of the proper results of the model is conditioned by providing exact data of the traffic parameters, even though it cannot be guaranteed that computed levels are always equal to real ones. Local sound events have a clear influence on the total instantaneous noise level. The verification based on a series of real measurements increases the credibility of the simulation results. The possibility of sound sample recording by the monitoring station was found very useful, because it helps to exclude sound events not related to the traffic noise. The speed of the noise map calculation was investigated. The time needed for obtaining the results is linearly dependent on the engaged computational power. Employing the supercomputer allows for creating such maps in reasonable time. Thus, the experiments demonstrate the potential usefulness of the so called "cloud computing" concept employment for environmental noise monitoring and creating acoustic maps in urban areas.

Future work will focus on expanding the number of monitoring stations to form a system able to indicate noise threats in larger city areas and to help create automatic credible and frequently updated noise maps of urban agglomerations.

\section{Acknowledgments}

Computing was performed by the Academic Computer Centre in Gdansk (CI TASK). Data visualization algorithms and plots were obtained with the help of Mr. Paweł Kozielecki.

\section{References}

1. Acoustics - Attenuation of sound during propagation outdoors - Part 2: General method of calculation, International Standard ISO 9613-2: 2000, International Organization for Standardization, Geneva, Switzerland, (1990). 
2. Acoustics - Description, measurement and assessment of environmental noise - Part 1: Basic quantities and assessment procedures, International Standard ISO 9613-1: 2003, (1990), International Organization for Standardization, Geneva, Switzerland.

3. Barelds R., Nota R. (2002), Propagation paths and reflections, Harmonoise WP 3 Technical Report, Den Haag.

4. Batko W., Stępień B. (2009), Non-parametric methods of estimation of type A uncertainty of the environmental noise hazard indices, Archives of Acoustics, 34, 3, 295-303.

5. Chang F., Chen C.J., Lu C.J. (2004), A linear-time component-labeling algorithm using contour tracing technique, Journal of CVIU, 93, 206-220.

6. Comeagaa D., Lazarovicib B., Tachec G. (2007), Reverse engineering for noise maps with application for Bucharest city, INTER-NOISE 2007, 28-31 August, Istanbul, Turkey.

7. Czyzewski A., Kostek B., Kotus J. (2008), Multimedia Interactive Services in Intelligent Environments Advanced Tools and Methodologies, Chapter, edited by Tsihrintzis G.A. and Lakhmi J., 275-295, Springer Verlag, Heidelberg, Studies in Computational Intelligence, 120, Berlin.

8. Czyzewski A., Kotus J., Kostek B. (2007), Determining the noise impact on hearing using psychoacoustical noise dosimeter, Archives of Acoustics, 32, 2, 215-229.

9. Czyzewski A., Kotus J., Kostek B., Szczodrak M. (2007), Multimedia Noise Monitoring System [in Polish: Multimedialny System Monitorowania Hałasu], Bezpieczeństwo Pracy, 7-8.2007, 8-11.

10. Czyzewski A., Kotus J., Kulesza M. (2005), Project and development of the automatic station for environmental noise monitoring [in Polish], ISSET 2005, Kraków, Poland, 53-60.

11. Dalka P. (2006), Detection and Segmentation of Moving Vehicles and Trains Using Gaussian Mixtures, Shadow Detection and Morphological Processing, Machine Graphics and Vision, 15, 339-348.

12. Directive 2002/49/EC of the European parliament and of the council of 25 June 2002 relating to the assessment and management of environmental noise, Official Journal of the European Communities, 2002.07.18, (2002).

13. Embleton T.F. (1996), Tutorial on sound propagation outdoors, J. Acoust. Soc. Am., 100, 1, 31-48.

14. ENGEL Z. (2001), Environmental protection against vibrations and noise [in Polish], PWN, Warszawa, Poland.

15. Engel Z. (2010), From the history of the noise control conference, Archives of Acoustics, 35, 2, 133-144.

16. Engel Z., ZaWieska W. (2004), Environmental noise protection, noise threat in the year of Poland joining EU [in Polish], 13th International Conf. on Noise Control, Gdynia 6-9.06 2004.

17. Furlanello C., Merler S., Menegon S., Mancuso S., Bertiato G. (2002), New WEBGIS technologies for geolocation of epidemiological data: an application for the surveillance of the risk of Lyme borreliosis disease, Proc. Spec. Issue Giornale Italiano di Aritmologia e Cardiostimolazione, 5, 1, 241-245. 
18. Golebiewsiki R., Makarewicz R. (2009), Verification of two methods of railway noise propagation, Archives of Acoustics, 34, 2, 177-188.

19. Harmon J.E., Anderson S.J. (2003), The Design and Implementation of Geographic Information Systems, John Wiley \& Sons, Inc., New Jersey.

20. Jonasson H., Sandberg U., van Blokland G., Ejsmont J., Watts G., Luminari M. (2004), Source modelling of road vehicles, Harmonoise Technical Report.

21. Kompala J., Lipowczan A. (2007), Noise hazard to the population of areas connected with functioning of roadway frontier crossings, Archives of Acoustics, 32, 2, 279-286.

22. Kotus J., Kostek B. (2008), The noise-induced harmful effect assessment based on the properties of the human hearing system, Archives of Acoustics, 33, 4, 435-440.

23. Kotus J., Szczodrak M., Czyzewski A., Kostek B. (2010), Long-term comparative evaluation of an acoustic climate in selected schools before and after the acoustic treatment, Archives of Acoustics, 35, 4, 551-564.

24. KuChARSKI R. (2007), Complex noise indicator for noise mapping based on the EU working groups' and Polish results of the annoyance investigations, Archives of Acoustics, 32, 2, 293-302.

25. Kulowski A. (1991), A modification of ray-tracing acoustics modeling method in rooms, Scientific Books of Gdansk University of Technology, 494, 1-117.

26. Li K.M., Taherzadeh S., Attenborough K. (1998), An improved ray-tracing algorithm for predicting sound propagation outdoors, J. Acoust. Soc. Am., 104, 4, 2077-2083.

27. NMPB-Routes-96, Road infrastructure noise - Calculation method including weather effects, CERTU, SETRA, LCPC, CSTB, January (1997).

28. Nota R., Barelds R., van MAercke D. (2005), Harmonoise WP 3 Engineering method for road traffic and railway noise after validation and fine-tuning, Harmonoise, Paris.

29. Pawelczyk M. (2008), Active noise control - a review of control-related problems, Archives of Acoustics, 33, 4, 509-520.

30. Popescu D.I., Moholea I.F. (2010), Monitoring the reaction and response of people to urban noise, Archives of Acoustics, 35, 2, 237-244.

31. Probst W. (2009), Strategic noise mapping - can it serve as a tool in city planning?, 16th Intern. Congress on Sound and Vibr., Kraków, 5-9 July.

32. Strategic Noise Maps for the city of Berlin, Germany, last access: 26.06.2008, http://www.stadtentwicklung.berlin.de/umwelt/umweltmap/ed705_01.htm\#A2.

33. Szczodrak M., Czyzewski A. (2008), Dynamic noise generation with an application of supercomputer, Scientific Books of Gdansk University of Technology, Informational Technologies, 16, 306-311.

34. Szczodrak M., Czyzewski A. (2009), Software for calculation of noise maps implemented on the supercomputer, Task Quarterly, 13, 3-4, 363-377.

35. Szczodrak M., Czyzewski A., Kotus J. (2008), Investigation of the road noise source employing an automatic noise monitoring station, Arch. of Acoust. Suppl., 33, 4, 77-83. 